\title{
Évaluation de l'adhésion aux directives nationales sur le dépistage et le diagnostic de l'infection par le VIH - Partie 2 : niveau de connaissance, aise et pratiques
}

\author{
GP Traversy'1, T Austin¹, J Yau¹, K Timmerman ${ }^{1 *}$
}

\section{Résumé}

Contexte : Le Guide pour le dépistage et le diagnostic de l'infection par le VIH (le Guide) de I'Agence de la santé publique du Canada (ASPC) propose des directives aux fournisseurs de soins de santé pour orienter le choix des personnes chez qui effectuer les tests de dépistage du $\mathrm{VIH}$, et pour décider à quel moment le faire et à quelle fréquence. Le dépistage et le diagnostic de l'infection par le VIH sont des étapes importantes pour atteindre les cibles 90-90-90 du Programme commun des Nations Unies sur le VIH/sida (ONUSIDA).

Objectif : Évaluer les niveaux de connaissance et d'aise des fournisseurs de soins de santé concernant différents aspects du dépistage du VIH, et déterminer si leurs pratiques de dépistage du VIH correspondent aux recommandations du Guide.

Méthodologie : Une enquête ouverte et anonyme, réalisée en ligne et comprenant des questions sur les connaissances, le niveau d'aise et les pratiques relativement au dépistage du $\mathrm{VIH}$, a été conçue avec la participation des intervenants, avant d'être validée et mise à l'essai. Elle a ensuite été transmise à un échantillon de commodité de fournisseurs de soins de santé d'un bout à l'autre du Canada, entre juin et août 2016.

Résultats : Au total, 1075 participants représentant l'ensemble des provinces et territoires canadiens ont répondu à l'enquête. La majorité des répondants étaient des membres du personnel infirmier (54\%) et des médecins (12\%). Dans l'ensemble, les connaissances sur le dépistage du VIH étaient bonnes, mais $37 \%$ des répondants ont sous-estimé le pourcentage de personnes au Canada qui vivent avec le $\mathrm{VIH}$ sans le savoir et seulement $32 \%$ des répondants savaient que les patients ont souvent des symptômes pendant une infection à VIH aiguë. La plupart des participants se sentaient à l'aise relativement au dépistage du VIH et environ $50 \%$ ont affirmé qu'ils offraient couramment le dépistage du VIH.

Conclusions : Dans l'ensemble, les connaissances et les pratiques correspondaient à ce qui est présenté dans le Guide pour le dépistage et le diagnostic de l'infection par le VIH de l'ASPC, mais certains fournisseurs de soins de santé peuvent sous-estimer le nombre de cas de VIH non diagnostiqués et mal interpréter les symptômes d'une infection à VIH aiguë. II faut interpréter ces résultats en tenant compte du fait qu'il s'agit d'un échantillon de commodité, mais ils laissent entendre qu'en comblant ces lacunes dans les connaissances, il pourrait être possible de diagnostiquer plus rapidement les infections au VIH chez ceux qui ne sont pas au courant de leur état.

\begin{abstract}
Affiliation
${ }^{1}$ Centre de la lutte contre les maladies transmissibles et les infections, Agence de la santé publique du Canada, Ottawa (Ontario)
\end{abstract}

${ }^{\star}$ Correspondance: karen. timmerman@canada.ca

Citation proposée : Traversy, G.P., T. Austin, J. Yau, et K. Timmerman. Évaluation de l'adhésion aux directives nationales sur le dépistage et le diagnostic de l'infection par le VIH - Partie 2 : niveau de connaissance, aise et pratiques. Relevé des maladies transmissibles au Canada. 2017;43(12):304-9. https://doi.org/10.14745/ccdr.v43i12a04f

\section{Introduction}

Le VIH est une maladie chronique qui peut toutefois être gérée au moyen de soins et de traitements adéquats. Cependant, selon des estimations réalisées en 2014, un peu plus d'une personne sur cinq au Canada est infectée par le VIH sans le savoir $(1,2)$. Cela signifie que ces personnes ne reçoivent pas les soins dont ils ont besoin et qu'elles transmettent peut-être le $\mathrm{VIH}$ à d'autres.
Les pratiques de dépistage et de diagnostic du VIH sont importantes, non seulement pour veiller à ce que les personnes atteintes soient aiguillées vers les soins et traitements adéquats, mais aussi pour parvenir à atteindre d'ici 2020 les cibles 90-90-90 du Programme commun des Nations Unies sur le $\mathrm{VIH} /$ sida (ONUSIDA) en vue de l'éradication du VIH. Ces cibles visent à ce que $90 \%$ des personnes vivant avec le $\mathrm{VIH}$ aient 
connaissance de leur infection, que $90 \%$ des personnes ayant reçu un diagnostic d'infection par le $\mathrm{VIH}$ reçoivent un traitement antirétroviral et que $90 \%$ des personnes recevant un traitement aient une charge virale supprimée (3).

En 2013, I'Agence de la santé publique du Canada (ASPC) a publié le Guide pour le dépistage et le diagnostic de l'infection par le VIH (le Guide) afin d'appuyer les efforts des fournisseurs de soins de santé qui procèdent au dépistage du VIH. Le Guide fait partie d'un éventail de produits que les fournisseurs de soins de santé peuvent utiliser. On y trouve des recommandations fondées sur des données probantes pour orienter le choix des personnes chez qui effectuer les tests de dépistage du VIH, et pour décider à quel moment le faire et à quelle fréquence. II propose également de l'information générale sur les procédures de dépistage et de counseling. Plus précisément, il recommande que les fournisseurs de soins de santé discutent du dépistage du VIH dans le cadre des soins médicaux de routine pour lutter contre la stigmatisation associée au dépistage et détecter les cas d'infection parmi la population à faible risque.

Au Canada, quelques études ont été réalisées pour cerner les tendances relativement aux pratiques de dépistage du $\mathrm{VIH}$ par les médecins et les autres fournisseurs de soins de santé, mais on ne sait pas dans quelle mesure les pratiques cliniques des fournisseurs de soins de santé correspondent aux recommandations de l'ASPC en matière de dépistage, ni dans quelle mesure ils se sentent à l'aise de suivre ces recommandations (4-6). À ce jour, l'ASPC n'a pas mené d'évaluation sur l'adoption des directives afin de déterminer à quel point les fournisseurs de soins de santé connaissent bien les recommandations du Guide pour le dépistage du VIH.

Le présent article décrit les résultats de la deuxième partie d'une étude plus large visant à évaluer le niveau d'adoption du Guide. L'objectif de la première partie consistait à déterminer si les fournisseurs de soins de santé savent que le Guide existe, s'ils I'utilisent et s'ils le trouvent utile (7). La deuxième partie avait pour objectif de déterminer si les fournisseurs de soins de santé connaissent les procédures de dépistage du VIH et s'ils sont à l'aise avec ces procédures, ainsi que d'évaluer les pratiques cliniques adoptées. L'étude dans son ensemble est réalisée dans le cadre des travaux visant à éclairer les mises à jour éventuelles du Guide en vue d'appuyer les activités de dépistage et de diagnostic de l'infection par le VIH au Canada.

\section{Méthodologie}

Les données concernant le niveau de connaissance du Guide, le niveau d'aise relativement à son contenu et les pratiques cliniques ont été recueillies sur une période de plus de trois mois (de juin à août 2016) dans le cadre d'une enquête anonyme plus vaste réalisée en ligne. Le cas échéant, la liste de vérification pour la déclaration des résultats d'enquêtes menées en ligne (Checklist for Reporting Results of Internet E-Surveys) a été employée pour la déclaration de la méthodologie et des résultats (8). Cette étude a reçu l'approbation du Comité d'éthique de la recherche de Santé Canada et de I'ASPC.

\section{Conception de l'enquête}

On a conçu cette enquête en consultation avec des spécialistes en matière d'évaluation, de maladies infectieuses et de $\mathrm{VIH}$. Le processus de conception s'est appuyé sur des enquêtes antérieures réalisées par l'ASPC avec des objectifs semblables, sur de la documentation consultée au préalable au sujet de la conception d'enquêtes, sur les facteurs qui affectent les comportements relatifs aux tests de dépistage, ainsi que sur les facteurs et les obstacles concernant la réalisation des tests (6,9-12). Un médecin spécialiste des maladies infectieuses et un spécialiste de l'évaluation ont procédé à un examen par des pairs externes du protocole régissant l'enquête et l'étude. Le questionnaire a ensuite été mis à l'essai auprès d'un groupe de spécialistes des maladies infectieuses avant sa diffusion à grande échelle.

Les connaissances des fournisseurs de soins de santé ont été évaluées de deux moyens. En premier lieu, les participants devaient répondre vrai ou faux à huit énoncés sur le VIH et le dépistage du VIH. Ensuite, les participants devaient indiquer à qui ils devraient proposer le dépistage du VIH parmi cinq groupes de patients : les personnes qui demandent à subir un test de dépistage du $\mathrm{VIH}$; les personnes qui présentent les symptômes et les signes d'un système immunitaire affaibli; les personnes actives sexuellement qui n'ont jamais subi le test; les personnes qui partagent du matériel de consommation de drogues avec un partenaire séropositif ou dont l'état sérologique est inconnu; les femmes enceintes ou qui prévoient le devenir et leur partenaire.

Le niveau d'aise des fournisseurs de soins de santé à l'égard des différents aspects du processus de dépistage a été évalué au moyen d'une question ouverte: «Dans quelle mesure êtes-vous à l'aise de discuter du VIH en général avec vos patients, notamment des facteurs de risque, du counseling avant et après le test, des résultats de test et de la conformité aux exigences de déclaration? ". Les réponses étaient codées séparément par deux personnes selon une échelle de cinq points (très à l'aise, à l'aise, relativement à l'aise, pas très à l'aise et pas du tout à l'aise). Des discussions permettaient ensuite de concilier les écarts.

Pour évaluer si les pratiques cliniques des fournisseurs de soins de santé correspondaient aux recommandations du Guide, on a demandé aux participants d'indiquer le pourcentage de leurs patients à qui ils proposaient un test de dépistage du VIH dans le cadre des rencontres de routine et à quelle fréquence ils abordaient six sujets (le dépistage du VIH, la fenêtre sérologique, les questions de confidentialité et de vie privée, les méthodes permettant de réduire les risques, les exigences relatives à la déclaration des cas positifs et les aiguillages vers les services de soutien en matière de $\mathrm{VIH}$ en cas de résultat positif) lors des séances de counseling sur le VIH avant et après un test de dépistage. On a aussi demandé aux participants d'indiquer, parmi une série d'énoncés sur les soins ou le counseling offerts de routine, lesquels étaient applicables dans leur cas. Il est possible d'obtenir sur demande des précisions supplémentaires sur ces variables et sur l'enquête dans son ensemble. 


\section{Recrutement et réalisation}

On a recruté des participants au moyen de bulletins électroniques et de listes de distribution, du site Web du Réseau canadien d'info-traitements sida (CATIE) et d'invitations par courriel transmises par des personnes-ressources d'autres ministères et bureaux régionaux du gouvernement du Canada. On a également envoyé un lien vers l'enquête à 23 associations de fournisseurs de soins de santé (comme des médecins, du personnel infirmier, des travailleurs sociaux et des fournisseurs de services communautaires). Bien que seulement trois associations professionnelles aient accepté de diffuser l'invitation à l'enquête (le Pacific AIDS Network, I'Association canadienne de santé publique et la Société canadienne du sida), il est possible que $\mathrm{d}^{\prime}$ autres associations l'aient transmise à leurs membres sans en informer l'équipe de recherche. Les personnes qui ont reçu I'invitation à l'enquête par courriel ou bulletin peuvent aussi I'avoir transmise à des collègues ou parmi leurs réseaux. II est donc impossible de calculer le taux de participation.

L'enquête était accessible en français et en anglais au moyen de l'outil d'enquête en ligne du Réseau canadien de renseignements sur la santé publique. On a demandé aux participants de fournir leur consentement éclairé pour la participation à l'enquête après leur avoir donné de l'information sur la gestion de la confidentialité, la gestion et le stockage des données, ainsi que la durée et le but de l'enquête, accompagnée des coordonnées du chercheur principal. Par ailleurs, les participants avaient le choix de répondre ou non à chacune des questions (sauf celles sur le consentement et pour la présélection). Aucun incitatif n'a été offert pour leur participation. Les réponses des participants ont été prises en compte s'ils avaient 18 ans ou plus, s'ils exerçaient à l'heure actuelle et s'ils étaient des professionnels de la santé ou des fournisseurs de soins de santé.

\section{Analyse et gestion des données}

On a recueilli les réponses à l'enquête dans une base de données sécurisée, puis elles ont été téléchargées dans un fichier Microsoft Excel protégé par mot de passe. Les réponses étaient anonymes; aucun identificateur personnel n'a été recueilli (p. ex., noms, adresses, courriels ou adresses IP). On a employé des statistiques descriptives pour résumer les caractéristiques de l'échantillon et les réponses aux questions de l'enquête. Les analyses ont été réalisées à l'aide de Microsoft Excel.

\section{Résultats}

Au total, 1075 fournisseurs de soins de santé provenant des 13 provinces et territoires ont répondu à l'enquête (tableau 1). Parmi les répondants à l'enquête, on a relevé notamment des membres du personnel infirmier (53,9\%), des médecins $(11,9 \%)$ et des travailleurs en santé communautaire $(8,9 \%)$, mais il y avait aussi du personnel infirmier praticien $(7,8 \%)$, des travailleurs sociaux (4,9\%), des personnes offrant du counseling $(3,6 \%)$ et des sages-femmes (3\%). Bon nombre des répondants travaillaient dans le domaine des infections transmissibles sexuellement et de la santé publique $(43,1 \%)$, dans un grand centre de population urbain $(53,2 \%)$ et comptaient plus de 20 ans d'expérience (38,3\%).
Tableau 1 : Caractéristiques démographiques des participants à l'enquête - Canada, de juin à août 2016*

\begin{tabular}{|c|c|c|}
\hline Caractéristiques démographiques & $\mathbf{n}$ & $\%$ \\
\hline \multicolumn{3}{|l|}{ Province ou territoire d'exee $(n=1,069)$} \\
\hline Ontario & 375 & 35.1 \\
\hline Colombie-Britannique & 152 & 14.2 \\
\hline Québec & 149 & 13.9 \\
\hline Saskatchewan & 107 & 10.0 \\
\hline Manitoba & 91 & 8.5 \\
\hline Alberta & 79 & 7.4 \\
\hline Nourveau-Brunswick & 30 & 2.8 \\
\hline Nouvelle-Écosse & 29 & 2.7 \\
\hline Terre-Neuve-et-Labrador & 22 & 2.1 \\
\hline Territoires du Nord-Ouest & 14 & 1.3 \\
\hline Île-du-Prince-Édouard & 11 & 1.1 \\
\hline Yukon & 7 & 0.7 \\
\hline Nunavut & 3 & 0.3 \\
\hline \multicolumn{3}{|l|}{ Type of provider $(n=1,071)$} \\
\hline Personnel infirmier & 577 & 53.9 \\
\hline Physician & 127 & 11.9 \\
\hline Community health worker & 95 & 8.9 \\
\hline Personnel infirmier practicien & 84 & 7.8 \\
\hline Travailleur social & 52 & 4.9 \\
\hline Personne offrant du conseil & 39 & 3.6 \\
\hline Safe-femme & 8 & 0.7 \\
\hline Médecin résident & 0 & 0.0 \\
\hline Autre fournisseur de soins de santé & 89 & 8.3 \\
\hline \multicolumn{3}{|l|}{ Domaine d'exercice $(n=1,055)$} \\
\hline $\begin{array}{l}\text { Infections transmissibles sexuellement et santé } \\
\text { publique }\end{array}$ & 455 & 43.1 \\
\hline Médecine familiale ou générale & 173 & 16.4 \\
\hline Spécialiste & 114 & 10.8 \\
\hline Soin d'urgence & 27 & 2.6 \\
\hline Autre (préciser) & 286 & 27.1 \\
\hline \multicolumn{3}{|l|}{ Milieu $(n=1,061)$} \\
\hline $\begin{array}{l}\text { Grand centre de population urbain }(100,000 \\
\text { habitants et plus) }\end{array}$ & 564 & 53.2 \\
\hline $\begin{array}{l}\text { Centre de population moyen (de 30,000 à 99,999 } \\
\text { habitants) }\end{array}$ & 181 & 17.1 \\
\hline $\begin{array}{l}\text { Petit centre de population (de 1,000 à } 29,999 \\
\text { habitants) }\end{array}$ & 234 & 22.1 \\
\hline Région rurale (moins de 1,000 habitants) & 62 & 5.8 \\
\hline $\begin{array}{l}\text { Région géographiquement isolée ou éloignée } \\
\text { (non accessible par route ou seulement accessible } \\
\text { par route d'hiver ou route de terre) }\end{array}$ & 20 & 1.9 \\
\hline \multicolumn{3}{|l|}{ Nombre d'années d'exercice $(n=1,061)$} \\
\hline Plus de 20 ans & 409 & 38.3 \\
\hline De 15 à 19 ans & 141 & 13.2 \\
\hline De 10 à 14 ans & 149 & 13.9 \\
\hline De 5 à 9 ans & 177 & 16.6 \\
\hline Moins de 5 ans & 193 & 18.1 \\
\hline
\end{tabular}

Page 306 RMTC • Le 7 décembre 2017 • Volume 43-12 


\section{Connaissances relativement au dépistage du $\mathrm{VIH}$}

Pour les questions de type vrai ou faux, plus de $90 \%$ des participants savaient que la présence d'autres maladies transmises sexuellement peut accroître le risque de transmission du VIH d'une personne, et que les personnes ayant des pratiques à risque élevé devraient subir un test de dépistage du VIH au moins une fois par année (figure 1). De 75 à $80 \%$ des répondants environ savaient qu'à la suite d'une exposition connue, il faut procéder à des tests lors du bilan initial et lors d'un suivi, qu'il n'est pas nécessaire d'obtenir un consentement écrit pour procéder à un test de dépistage et que l'existence de facteurs de risque n'est pas nécessaire pour faire passer un test. Environ le tiers des répondants $(32,4 \%)$ seulement savaient que les patients présentent souvent des symptômes pendant une infection à $\mathrm{VIH}$ aiguë et plus du tiers des répondants $(37,1 \%)$ estimaient que le taux de VIH non diagnostiqué au Canada était de $10 \%$ ou moins, alors que le taux réel s'établit plutôt à $21 \%$ (2). Dans la question sur les profils de patients, plus de $91 \%$ des répondants ont indiqué correctement que les cinq groupes de patients devraient se voir offrir un test de dépistage du VIH (données non présentées).

Figure 1 : Pourcentage des répondants qui ont répondu correctement aux questions de type vrai ou faux $(n=1059$ à 1068$)$

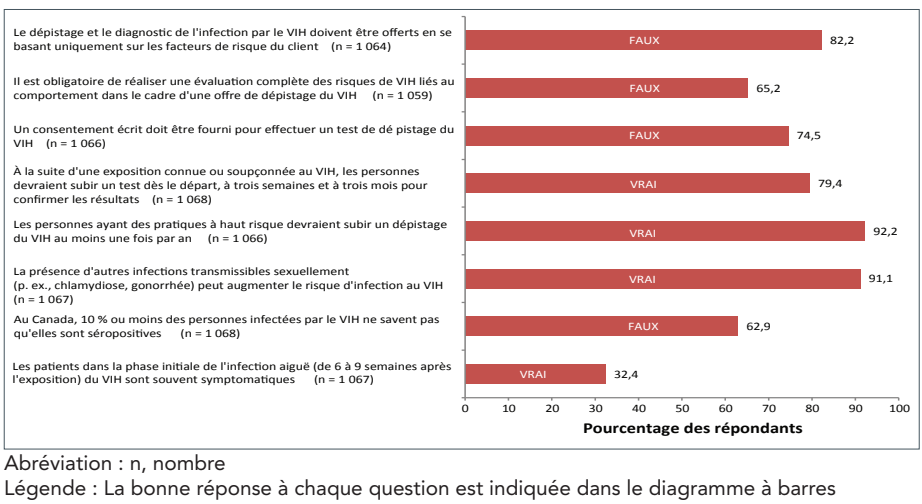

\section{Niveau d'aise relativement aux différents aspects de la procédure de dépistage du VIH}

Lorsqu'on a demandé aux répondants dans quelle mesure ils se sentent à l'aise de discuter du VIH avec leurs patients (notamment des facteurs de risque, du counseling avant et après un test de dépistage, de la communication des résultats et du respect des exigences de déclaration), un peu plus de la moitié $(55,9 \%)$ ont affirmé être très à l'aise relativement aux différents aspects de la procédure de dépistage du VIH. Seulement 6,0 \% des répondants ont indiqué être plutôt ou très mal à l'aise au sujet du dépistage du VIH (tableau 2).

\section{Pratiques cliniques relativement au dépistage du VIH}

Plus de la moitié des répondants $(52,6 \%)$ ont déclaré offrir le dépistage du $\mathrm{VIH}$, et $41,2 \%$ ont affirmé proposer le dépistage du VIH lorsqu'ils offrent le dépistage d'autres infections
Tableau 2 : Nombre et pourcentage de participants ayant indiqué les différents niveaux d'aise pour ce qui est de discuter du VIH avec leurs patients $(n=947)$

\begin{tabular}{|l|r|r|}
\hline $\begin{array}{l}\text { Niveau d'aise pour ce qui est de discuter du VIH } \\
\text { avec les patients }\end{array}$ & $\mathbf{n}$ & $\%$ \\
\hline Très ou tout à fait à l'aise & 529 & 55,9 \\
\hline À l'aise & 187 & 19,8 \\
\hline Relativement à I'aise & 119 & 12,6 \\
\hline Pas très à l'aise & 31 & 3,3 \\
\hline Pas du tout à l'aise & 24 & 2,5 \\
\hline Ne s'applique pas & 57 & 6 \\
\hline
\end{tabular}

Abréviation : $n$, nombre

Remarque : Niveau d'aise pour ce qui est de discuter du VIH avec les patients, notamment des facteurs de risque, du counseling avant et après un test de dépistage, de la communication des résultats et du respect des exigences de déclaration

transmissibles sexuellement (figure 2). À l'opposé, près du quart des participants $(24,1 \%)$ ont déclaré offrir des tests de routine à moins de $25 \%$ de leurs patients, voire à aucun patient.

Figure 2 : Pourcentage des répondants qui ont indiqué que les énoncés suivants s'appliquent à eux $(n=1075)$

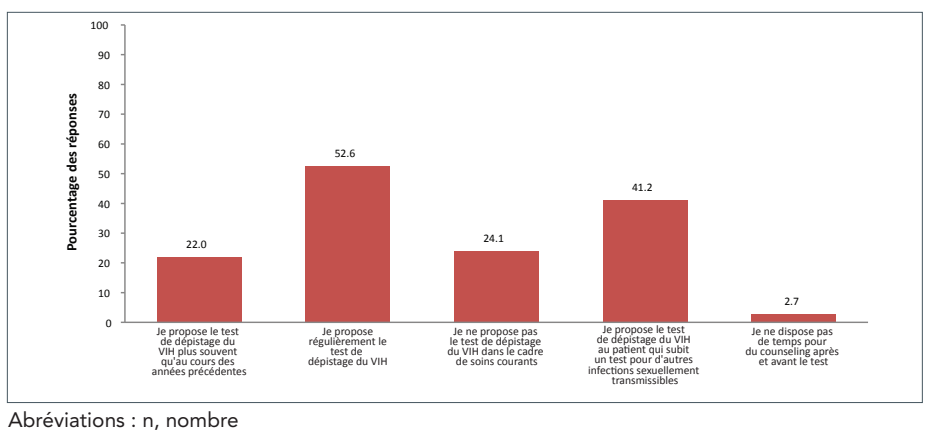

Abréviations : n, nombre

Lorsqu'on leur a demandé s'ils proposent le dépistage du VIH dans le cadre des soins de routine, $48,6 \%$ des répondants ont affirmé l'offrir de 75 à $100 \%$ du temps (tableau 3).

Tableau 3 : Pourcentage des patients qui se voient offrir le dépistage du VIH dans le cadre des soins de routine $(n=621)$

\begin{tabular}{|l|r|r|}
\hline $\begin{array}{c}\text { À quel pourcentage des patients offrez-vous } \\
\text { le dépistage du VIHdans le cadre des soins de } \\
\text { routine (examens médicaux ou annuels) }\end{array}$ & $\mathbf{n}$ & $\%$ \\
\hline De 75 à $100 \%$ & 302 & 48,6 \\
\hline De 50 à $75 \%$ & 100 & 16,1 \\
\hline De 25 à $50 \%$ & 57 & 9,2 \\
\hline Moins de $25 \%$ & 83 & 13,4 \\
\hline $\begin{array}{l}\text { Je n'offre jamais le dépistage du VIH de routine à mes } \\
\text { patients }\end{array}$ & 79 & 12,7 \\
\hline
\end{tabular}

Abréviation : n, nombre 


\section{Discussion}

Dans l'ensemble, les résultats de cette enquête nationale suggèrent que les fournisseurs de soins de santé ont une bonne connaissance de la procédure de dépistage du VIH et qu'ils sont à l'aise avec cette procédure, mais qu'il y a quelques lacunes dans les connaissances. Les deux aspects lacunaires dans les connaissances (le pourcentage sous-estimé de personnes vivant avec le VIH sans le savoir et le fait que les patients atteints du VIH présentent souvent des symptômes pendant la période d'infection aiguë), ainsi que le fait que la moitié des participants ont déclaré ne pas offrir le dépistage du VIH dans le cadre des soins de routine, pourraient faire que l'on manque des occasions de diagnostiquer le VIH à un stade précoce. L'absence de risque perçu a déjà été signalée comme un obstacle important au dépistage du VIH (11).

Parmi les forces de la présente étude, mentionnons l'échantillon géographiquement représentatif, avec des répondants provenant de toutes les provinces et tous les territoires, ainsi que la diversité des fournisseurs de soins de santé. En outre, l'enquête était exhaustive et incluait plusieurs aspects qui pourraient servir lors de la mise à jour du Guide.

Quant à ses limitations, soulignons le recours à un échantillon de commodité, ce qui fait que les réponses des participants ne sont peut-être pas représentatives de l'ensemble des fournisseurs de soins de santé qui offrent le dépistage du VIH au Canada. Certains de nos modes de distribution pourraient avoir été plus susceptibles de cibler les fournisseurs de soins de santé dans le domaine de la santé sexuelle, qui connaissent mieux que d'autres la question du dépistage du VIH et qui sont plus à l'aise avec cette question. En outre, le recours à l'autoévaluation pour évaluer les pratiques cliniques touchant le dépistage du VIH pourrait avoir entraîné un biais de rappel dans le cadre de cette étude.

\section{Conclusion}

Le dépistage du VIH est la première étape en vue d'entamer un traitement pour le VIH permettant d'améliorer la vie des personnes vivant avec cette infection et leurs partenaires, ainsi que pour atteindre les cibles 90-90-90 de I'ONUSIDA. Les résultats de cette enquête pourraient servir à éclairer la rédaction des futures versions du Guide ainsi que I'élaboration $d$ 'autres initiatives de transfert des connaissances pour améliorer les niveaux de connaissances et d'aise des fournisseurs de soins de santé relativement au dépistage du $\mathrm{VIH}$, dans le cadre des efforts globaux d'élimination du VIH au Canada.

\section{Déclaration des auteurs}

G.T. - Conceptualisation, méthodologie, collecte et stockage des données, analyse formelle, rédaction - ébauche originale, rédaction - examen et révision, supervision, administration du projet

T.A. - Méthodologie, collecte et stockage des données, rédaction - ébauche originale, rédaction - examen et révision J.Y. - Méthodologie, collecte et stockage des données, rédaction - ébauche originale, rédaction - examen et révision
K.T. - Conceptualisation, méthodologie, rédaction - ébauche originale, rédaction - examen et révision, supervision, administration du projet

\section{Conflit d'intérêt}

Aucun.

\section{Contributions}

Les auteurs aimeraient remercier les personnes suivantes (sans ordre particulier) pour leur contribution à ce manuscrit :

Kelsey Young - Analyse formelle Shalane $\mathrm{Ha}$ - Méthodologie, rédaction - examen et révision Jun Wu - Méthodologie, rédaction - examen et révision, administration du projet

Margaret Gale-Rowe - Méthodologie, rédaction - examen et révision, administration du projet

Makenzie Weekes - Rédaction - examen et révision

Priya Prabhakar - Rédaction - examen et révision

Militza Zencovich - Méthodologie

Kanchana Amaratunga - Méthodologie

Courtney Smith - Analyse formelle

Mandip Maheru - Méthodologie

Ulrick Auguste - Méthodologie

Katie Freer - Méthodologie

Dena Schanzer - Méthodologie, analyse formelle

Mary Lysyk - Méthodologie

Katherine Dinner - Méthodologie

Simon Foley - Conceptualisation, méthodologie

\section{Remerciements}

Les auteurs aimeraient souligner les contributions reçues de diverses personnes tout au long des différentes étapes du projet pour la conception de l'enquête, les soumissions au Comité d'éthique de la recherche, la collecte des données, l'analyse et l'interprétation. Ils tiennent à remercier l'équipe du Réseau canadien de renseignements sur la santé publique de les avoir aidés à se servir de l'outil d'enquête, ainsi que le Comité d'éthique de la recherche de Santé Canada. Ils souhaitent remercier également le groupe de travail d'experts sur les Lignes directrices canadiennes sur les infections transmissibles sexuellement pour leur aide lors de la mise à l'essai de l'enquête, sans oublier les diverses organisations qui ont aidé à diffuser l'enquête.

\section{Financement}

Les auteurs n'ont aucune source externe de financement à déclarer. Cette étude était soutenue par l'Agence de la santé publique du Canada.

\section{Références}

1. Agence de la santé publique du Canada. Virus de l'immunodéficience humain: Guide pour le dépistage et le diagnostic de l'infection par le VIH. Ottawa; 2013. http:// 
www.catie.ca/sites/default/files/FR_Guide-pour-le-depistageet-le-diagnostic-de-linfection-par-le-VIH-2013.pdf

2. Agence santé publique du Canada. Résumé : Estimations de l'incidence de la prévalence, et de la proportion non diagnostiquée au VIH au Canada, 2014. Ottawa; 2015. https://www.canada.ca/fr/sante-publique/services/ publications/maladies-et-affections/resume-estimationsincidence-prevalence-et-proportion-non-diagnostiquee-vihcanada-2014.html

3. Programme commun des National Unies sur le VIH/sida (ONUSIDA). 90-90-90: Une cible ambitieuses de traitement pour aider à mettre fin à l'épidémie du sida. 2014. http:// www.unaids.org/fr/resources/documents/2014/90-90-90

4. Bungay $V$, Masaro CL, Gilbert M. Examining the scope of public health nursing practice in sexually transmitted infection prevention and management: what do nurses do? J Clin Nurs 2014 Nov;23(21-22):3274-85. DOl (http://dx.doi. org/10.1111/jocn.12578). PubMed (https://www.ncbi.nlm. nih.gov/entrez/query.fcgi?cmd=Retrieve $\& d b=$ PubMed\&li st_uids=24606574\&dopt=Abstract).

5. MacDonald SE, Hartling LA, Seguin RM, O'Connor KS, Rekart ML, Mowat DL et al. Screening for HIV during pregnancy. Survey of physicians' practices. Can Fam Physician 2001 Nov;47:2250-7. PubMed (https://www.ncbi. nlm.nih.gov/entrez/query.fcgi?cmd=Retrieve\&db=PubMed\&l ist_uids=11768923\&dopt=Abstract).

6. Rank C, Remis RS, Swantee C, Wu K. Patterns of HIV testing among Ontario physicians. Can J Public Health 2010 JulAug;101(4):294-9. PubMed (https://www.ncbi.nlm.nih. gov/entrez/query.fcgi?cmd=Retrieve\&db=PubMed\&lis t_uids=21033534\&dopt=Abstract)

7. Traversy G, Austin T, Yau J, Timmerman K. Évaluation de I'adhésion aux directives nationales sur le dépistage et le diagnostic de l'infection par le VIH - Partie 1 : connaissance, utilisation et utilité. Relevé des maladies transmissibles au Canada. 2017;43(12):298-303. https://www.canada. $\mathrm{ca} / \mathrm{fr} /$ sante-publique/services/rapports-publications/ releve-maladies-transmissibles-canada-rmtc/numeromensuel/2017-43/rmtc-volume-43-12-7-decembre-2017/ diagnostic-infection-vih-partie-1.html

8. Eysenbach G. Improving the quality of Web surveys: the Checklist for Reporting Results of Internet E-Surveys (CHERRIES). J Med Internet Res 2004 Sep;6(3):e34. DOI (http://dx.doi.org/10.2196/jmir.6.3.e34). PubMed (https:// www.ncbi.nlm.nih.gov/entrez/query.fcgi?cmd=Retrieve $\& d b=$ PubMed\&list_uids=15471760\&dopt=Abstract).

9. Smith CR, Pogany L, Foley S, Wu J, Timmerman K, GaleRowe $\mathrm{M}$ et al. Knowledge and counselling practices of Canadian physicians related to antibiotic use and antimicrobial resistance: A two-cycle national survey in 2014/2015. Can Fam Physician. Sous presse.

10. Skinner K. Developing a tool to measure knowledge exchange outcomes. Can J Program Eval 2007;22(1):49-73.

11. Traversy G, Austin T, Ha S, Timmerman K, Gale-Rowe M. Un aperçu des récentes données probantes sur les obstacles et les facteurs favorables au dépistage du VIH. Relevé des maladies transmissible au Canada. 2015;41(12):445-63. https://www.canada.ca/content/dam/phac-aspc/migration/ phac-aspc/publicat/ccdr-rmtc/15vol41/dr-rm41-12/assets/ pdf/15vol41_12-fra.pdf

12. Seto J, Kapral O, Wong T, Gale-Rowe M, Demers A, Dodds $J$ et al. Differences among Canadian family practitioners by years of practise in uptake of sexually transmitted and blood-borne infections (STBBIs) clinical recommendations (STI\&AIDS World Congress 2013 Vienna, Austria). Sex Transm Infect 2013 Jul;89 Suppl 1:A39. DOI (http://dx.doi. org/10.1136/sextrans-2013-051184.1225) 